Annales Academiæ Scientiarum Fennicæ

Series A. I. Mathematica

Volumen 2, 1976, 113-127
Commentationes in honorem

Rolf Nevanlinna

LXXX annos nato

\title{
FINELY HARMONIC MAPPINGS AND FINELY HOLOMORPHIC FUNCTIONS
}

\author{
BENT FUGLEDE
}

Introduction. The important paper by Constantinescu and Cornea [2] on compactifications of harmonic spaces contains an interesting study of harmonic mappings between two such spaces $\Omega$ and $\Omega^{\prime}$, that is, morphisms with respect to the harmonic structures on $\Omega$ and $\Omega^{\prime}$, see $[2, \S 3]$.

The principal result concerning such harmonic mappings is $[2$, Theorem 3.5], according to which every non-constant harmonic mapping of $\Omega$ (or of a domain $X \subset \Omega$ ) into $\Omega^{\prime}$ is an open mapping, not necessarily in the usual (= initial) topologies on $\Omega$ and $\Omega^{\prime}$, but in the fine topology on these spaces (i.e., the coarsest topology making all superharmonic functions continuous).

In view of this result it seems natural to ask whether the theory of harmonic mappings could be extended so as to allow the above domain $X$ to be replaced by any fine domain (i.e., a finely open and finely connected set) in $\Omega$. This will represent a true generalization since the fine topology on a harmonic space is (in general strictly) stronger than the initial topology.

We are thus led to study the notion of a finely harmonic mapping $\varphi: X \rightarrow \Omega^{\prime}$, where $X$ denotes a fine domain in $\Omega$. Thus $\varphi$ should be a morphism with respect to the fine harmonic structure on $\Omega$ and $\Omega^{\prime}$. This structure was introduced and studied in [6] under the crucial hypothesis that the harmonic space in question satisfies the domination axiom $D$ (as is the case, e.g., for Riemann surfaces or Riemannian manifolds).

The first study of finely harmonic mappings was made by Laine [8], who however confined himself to the case where $X$ is open in the initial topology on $\Omega$ (or equivalently: the case $X=\Omega$ ), in other words the same case as in [2].

Our main result, given in Theorem 7 and Theorem 6 below, asserts that (if the points of $\Omega^{\prime}$ are polar) every non-constant finely harmonic mapping of a fine domain $X \subset \Omega$ into $\Omega^{\prime}$ has a finely open range and determines a finely open mapping. Moreover, the pre-image of any polar subset of 
$\Omega^{\prime}$ is polar in $\Omega$. In particular, such a mapping cannot be constant in any non-void finely open subset of its domain of definition, $X$.

In the particular case where $\Omega=\Omega^{\prime}=\mathbf{C}$ (the complex plane) this result applies, in particular, to the finely holomorphic functions, and here it reproduces a result recently obtained by Debiard and Gaveau [4] by a quite different method involving stochastic differentiation along Brownian paths, while the present paper solely uses methods of harmonic spaces. After seeing the present paper in manuscript, Debiard and Gaveau have informed me that their method likewise applies to the study of finely harmonic mappings, thus allowing for an alternative proof of Theorems 6 and 7 below.

1. Hypotheses and a key lemma. Throughout the present paper (except for the last section, $\S 11$ ) it is assumed that $\Omega$ and $\Omega^{\prime}$ are harmonic spaces in the sense of Constantinescu and Cornea [3] with a countable base and satisfying the domination axiom $D$, see [3, Chapter 9$].{ }^{1}$

Among the consequences of axiom $D$ recall that the regular domains in such a space form a base for the given (= initial) topology [3, Corollary 9.2.4], that polar and semipolar sets are the same [3, Corollary 9.2.3], and that the fine topology is locally connected [6, Corollary 9.11], [3, Exercise 9.2.4].

The decisive tool which allows us to carry over the methods of Constantinescu and Cornea in $[2, \S 3]$ to the present general case of a finely harmonic mapping defined on a fine domain is the following lemma (a special case of which is implicit in $[7, \S 4]$ ). This lemma also enters in the work of Debiard and Gaveau (to whom I had communicated it along with a slightly different proof).

$\mathrm{L}$ e $\mathrm{m} \mathrm{m}$ a. Let $\varphi: X \rightarrow Y$ be a finely continuous mapping of a finely open set $X \subset \Omega$ into a separable, metrizable, topological space $Y$ (e.g. $\left.Y=\Omega^{\prime}\right)$. Every point $x_{0} \in X$ has then a fine neighbourhood $K \subset X$ such that $K$ is compact and $\varphi \mid K$ is continuous in the initial topology on $\Omega$.

Proof. We may assume that $\Omega$ is $\mathfrak{B}$-harmonic, see [3, Theorem 2.3.3]. $Y$ can be imbedded as a subspace of $[0,1]^{\mathrm{N}}$, and hence it suffices to consider the case $Y=[0,1]^{\mathrm{N}}$. For any $x \in X$ we may then write

1 The fine harmonic structure was studied in [6] in the framework of a strong harmonic space $\Omega$ in the sense of Bauer, satisfying the domination axiom. Since Doob's convergence axiom is practically not used, but only the original convergence axiom of Bauer, the theory carries over to any $\mathfrak{B}$-harmonic space in the sense of Constantinescu and Cornea [3] with a countable base and satisfying axiom $D$, the only exception being part of $[6, \S 10.12-\$ 10.15]$. Since any harmonic space in the sense of [3] can be covered by $\mathfrak{B}$-harmonic spaces, the frame indicated above is adequate for local aspects of the theory of fine harmonicity. 


$$
\varphi(x)=\left(\varphi_{n}(x)\right)_{n \in \mathbf{N}},
$$

where each $\varphi_{n}$ is a finely continuous function on $X$ into $[0,1]$. Let $f_{n}$ and $g_{n}$ denote the extensions of $\varphi_{n}$ from $X$ to $\Omega$ obtained by putting $f_{n}=1, g_{n}=0$ in $\Omega \backslash X$. Then $f_{n}$ is finely upper and $g_{n}$ finely lower semicontinuous. According to Brelot [1, Theorem 7] the weight, or capacity,

$$
A \mapsto R_{1}^{A}\left(x_{0}\right)
$$

has the Choquet property. ${ }^{2}$ Hence there exists, by [1, Theorem 3] or $[5, \S 4.3]$, for any $n \in \mathbf{N}$, an open set $\omega_{n} \subset \Omega$ (in the initial topology on $\Omega$ ) such that $f_{n} \mid \Omega \backslash \omega_{n}$ is upper and $g_{n} \mid \Omega \backslash \omega_{n}$ is lower semicontinuous (in the initial topology), and such that moreover

$$
R_{1}^{\omega_{n}}\left(x_{0}\right)<2^{-n} .
$$

It is well known that there exists a compact, fine neighbourhood $V$ of $x_{0}$ such that $V \subset X$. Now write

$$
\omega=\bigcup_{n} \omega_{n}, \quad K=V \backslash \omega .
$$

Since

$$
R_{1}^{\omega}\left(x_{0}\right)<\sum 2^{-n}=1,
$$

$\omega$ is thin at $x_{0}$, and $x_{0} \notin \omega$, and so $K$ is a compact, fine neighbourhood of $x_{0}$ in $X$. Since $f_{n}=g_{n}=\varphi_{n}$ in $X$, it follows that each $\varphi_{n} \mid K$, and hence also $\varphi \mid K$, is continuous in the initial topology.

Remark. The lemma obviously extends to the case of a countable family of finely continuous mappings $\varphi_{n}: X \rightarrow Y_{n}$ into spaces $Y_{n}$ as above, and $K$ may be chosen as to work for all $\varphi_{n}$ simultaneously. (It suffices to consider the product mapping into the separable metric product space $\Pi_{n \in \mathrm{N}} Y_{n}$.)

2. Definition. $A$ mapping $\varphi: X \rightarrow \Omega^{\prime}$ (with $X$ finely open in $\Omega$ ) is called a finely harmonic mapping if the following two conditions are fulfilled:

i) $\varphi$ is continuous from $X$ with the fine topology to $\Omega^{\prime}$ with its initial topology.

ii) $u^{\prime} \circ \varphi$ is a finely harmonic function in $\varphi^{-1}\left(U^{\prime}\right)$ for any harmonic function $u^{\prime}$ in a usual open set $U^{\prime} \subset \Omega^{\prime} \quad\left(\right.$ such that $\left.\varphi^{-1}\left(U^{\prime}\right) \neq \varnothing\right)$.

For an alternative, equivalent definition see Theorem 5 below, where a finely harmonic mapping is characterized as a morphism with respect to the fine harmonic structure on both $\Omega$ and $\Omega^{\prime}$.

2 This result easily extends to the present case of a $\mathfrak{P}$-harmonic space $\Omega$ with a countable base (and satisfying axiom $D$ ). (Use [3, Theorem 9.2.1, h)] together with the method in [1].) 
3. Immediate consequences. a) For variable $X$, the finely harmonic mappings of $X$ into $\Omega^{\prime}$ have the sheaf property (with respect to the fine topology on $\Omega$ ).

This implies that, for any open set $\omega^{\prime} \subset \Omega^{\prime}$, the restriction of a finely harmonic mapping $\varphi: X \rightarrow \Omega^{\prime}$ to the finely open set $\varphi^{-1}\left(\omega^{\prime}\right)(\subset X)$ is a finely harmonic mapping of $\varphi^{-1}\left(\omega^{\prime}\right)$ into the space $\omega^{\prime}$ (and likewise into $\Omega^{\prime}$ ).

b) If $\Omega^{\prime}=\mathbf{R}$ (with the affine sheaf), then $\varphi: X \rightarrow \mathbf{R}$ is a finely harmonic mapping if and only if 1 and $\varphi$ are finely harmonic functions in $X$ (in the sense of [6]).

c) If $\Omega^{\prime}=\mathbf{C}$ (identified with $\mathbf{R}^{2}$ endowed with the classical harmonic sheaf), then $\varphi: X \rightarrow \mathbf{C}$ is a finely harmonic mapping if and only if $\varphi^{n}$ is a complex, finely harmonic function (i.e., has finely harmonic real and imaginary parts) for every $n=0,1,2, \ldots$ More generally:

d) If $\Omega^{\prime}=\mathbb{R}^{n^{\prime}}$ (with the classical harmonic sheaf), then $\varphi: X \rightarrow \mathbf{R}^{n^{\prime}}$ is a finely harmonic mapping if and only if $H^{\prime} \circ \varphi$ is a finely harmonic function in $X$ for every harmonic polynomial $H^{\prime}$ on $\mathbf{R}^{n^{\prime}}$. (Use [6, Lemma 9.6].)

e) If $X$ is open in $\Omega$ (e.g., $X=\Omega$ ), then every harmonic mapping $\varphi: X \rightarrow \Omega^{\prime}$ in the sense of [2] is a finely harmonic mapping (in the above sense). - The two notions are identical if, e.g., the points of $\Omega^{\prime}$ are polar, as shown by Laine [8, Theorem 2.1.5].

4. L e m m a. If $\varphi: X \rightarrow \Omega^{\prime}$ is a finely harmonic mapping, then $s^{\prime} \circ \varphi$ is a finely hyperharmonic function in $X$ for any superharmonic function $s^{\prime}$ on $\Omega^{\prime}$.

Proof. For any regular domain $V^{\prime}$ in $\Omega^{\prime}$ write

$$
s_{V^{\prime}}^{\prime}:=\left\{\begin{array}{lll}
s^{\prime} & \text { in } & \Omega^{\prime} \backslash V^{\prime} \\
H_{s^{\prime}}^{V^{\prime}} & \text { in } & V^{\prime}
\end{array}\right.
$$

Then $s_{V^{\prime}}^{\prime}$ is superharmonic and $\leqq s^{\prime}$ in $\Omega^{\prime}$, and harmonic in $V^{\prime}$.

According to Lemma 1, every point $x \in X$ has a compact, fine neighbourhood $K \subset X$ such that $\varphi \mid K$ is continuous in the initial topologies on $K$ and $\Omega^{\prime}$, and hence $\varphi(K)$ is compact. We shall prove that $s^{\prime} \circ \varphi$ is finely hyperharmonic in the fine interior of $K$.

Following [2,p. $20 \mathrm{f}$.], we denote by $\mathfrak{S}^{\prime}$ any finite covering of $\varphi(K)$ by regular domains $V^{\prime}$ in $\Omega^{\prime}$, and write

$$
s_{\mathfrak{Y}}^{\prime}:=\min _{V^{\prime} \in \mathfrak{S}^{\prime}} s_{V^{\prime}}^{\prime}
$$


Then $s_{\mathfrak{B}^{\prime}}^{\prime}$ is superharmonic and $\leqq s^{\prime}$ in $\Omega^{\prime}$. Our main task is to prove that $s_{\mathfrak{S}^{\prime}}^{\prime}{ }^{\circ} \varphi$ is finely hyperharmonic in the fine interior of $K$.

In view of the open problem raised in $[6, \S 8.5]$ we must now deviate from the reasoning in [2]. Since the finely open sets $\varphi^{-1}\left(V^{\prime}\right)$ cover $K$ when $V^{\prime}$ ranges over $\mathfrak{B}^{\prime}$, it suffices to prove that $s_{\mathfrak{S}}^{\prime}{ }^{\prime} \circ \varphi$ is finely hyperharmonic in

$$
U:=\varphi^{-1}\left(U^{\prime}\right)
$$

for any prescribed $U^{\prime} \in \mathfrak{S}^{\prime}$.

We begin by proving that, for any other member $V^{\prime}$ of $\mathfrak{S}^{\prime}$, the function

$$
s_{\left\{U^{\prime}, V^{\prime}\right\}}^{\prime} \circ \varphi=\min \left(s_{U^{\prime}}^{\prime} \circ \varphi, s_{V^{\prime}}^{\prime} \circ \varphi\right)
$$

is finely hyperharmonic in $U$, using the fact that $s_{U^{\prime}}^{\prime} \circ \varphi$ is finely hyperharmonic (even finely harmonic) in $U$ by Definition 2 because $s_{U^{\prime}}^{\prime}$ is harmonic in $U^{\prime}$. Similarly, $s_{V^{\prime}}^{\prime} \circ \varphi$ is finely harmonic in $\varphi^{-1}\left(V^{\prime}\right)$, in particular in $U \cap \varphi^{-1}\left(V^{\prime}\right)$, and the function (1) equals $s_{U^{\prime}}^{\prime} \circ \varphi$ in $U \backslash \varphi^{-1}\left(V^{\prime}\right)$. Since moreover $(1)$ is finely lower semicontinuous in $U$, it follows from [6, Lemma 10.1] that (1) is finely hyperharmonic in $U$.

Next it follows by the same argument that, for any third member $W^{\prime}$ of $\mathfrak{B}^{\prime}$, the function

$$
s_{\left\{U^{\prime}, V^{\prime}, W^{\prime}\right\}}^{\prime} \circ \varphi=\min \left(s_{\left\{U^{\prime}, V^{\prime}\right\}}^{\prime} \circ \varphi, s_{W^{\prime}}^{\prime} \circ \varphi\right)
$$

is finely hyperharmonic in $U$. In a finite number of steps we thus prove that $s^{\prime} \mathfrak{B}^{\prime}{ }^{\circ} \varphi$ is finely hyperharmonic in $U$, and therefore in the fine interior
of $K$.

The finite coverings $\mathfrak{B}^{\prime}$ of $\varphi(K)$ considered above form a directed set under the pre-order relation defined by

$$
\mathfrak{B}_{1}^{\prime} \leqq \mathfrak{B}_{2}^{\prime} \Leftrightarrow \forall V_{2}^{\prime} \in \mathfrak{B}_{2}^{\prime} \exists V_{1}^{\prime} \in \mathfrak{B}_{1}^{\prime}: V_{1}^{\prime} \supset V_{2}^{\prime} .
$$

The net $\left(s_{\mathfrak{B}}^{\prime}\right)$, indexed by this directed set, is known to be pointwise increasing to the limit

$$
s^{\prime}=\sup s_{\mathfrak{B}^{\prime}}^{\prime}
$$

Hence $s^{\prime} \circ \varphi$ is the pointwise limit of the increasing net of finely hyperharmonic functions $s_{\mathfrak{B}}^{\prime}{ }^{\circ} \varphi$ in the fine interior of $K$. Consequently, by [6, Corollary 2, p. 84], $s_{\mathfrak{B}^{\prime}}^{\prime}{ }^{\circ} \varphi$ is likewise finely hyperharmonic in the fine interior of $K$, and so actually in all of $X$. This completes the proof of the lemma. 
5. Th e or e m. A mapping $\varphi: X \rightarrow \Omega^{\prime}$ (with $X$ finely open in $\Omega$ ) is a finely harmonic mapping if and only if it has the following two properties:

i) $\varphi$ is continuous in the fine topologies on both $X$ and $\Omega^{\prime}$.

ii) $s^{\prime} \circ \varphi$ is a finely hyperharmonic function in $\varphi^{-1}\left(U^{\prime}\right)$ for any finely hyperharmonic function $s^{\prime}$ in a finely open set $U^{\prime} \subset \Omega^{\prime}$ (such that $\left.\varphi^{-1}\left(U^{\prime}\right) \neq \varnothing\right)$.

Proof. The "if part" is obvious in view of [6, Theorem 8.7]. In proving the "only if part", we may assume by virtue of [3, Theorem 2.3.3] that $\Omega^{\prime}$ is a $\mathfrak{\$}$-harmonic space, cf. the latter part of a), §3. Thus let $\varphi$ denote a harmonic mapping of $X$ into a $\mathfrak{P}$-harmonic space $\Omega^{\prime}$.

Since the fine topology on $\Omega^{\prime}$ is the coarsest one making all superharmonic functions $s^{\prime}$ on $\Omega^{\prime}$ continuous, Property i) will follow once we can show that $s^{\prime} \circ \varphi$ is finely continous in $X$ for every such $s^{\prime}$. And this is indeed the case by virtue of Lemma 4, according to which $s^{\prime} \circ \varphi$ is finely hyperharmonic in $X$, and hence finely continuous there [6, Theorem 9.10].

In proving ii), we may assume that $s^{\prime}<+\infty$ in $U^{\prime}$. (The reduction to this case can be performed by choosing a finite potential $q^{\prime}>0$ on $\Omega^{\prime}$ and representing $s^{\prime}$ as the pointwise limit of the increasing sequence of finite valued, finely hyperharmonic functions $\min \left(s^{\prime}, n q^{\prime}\right)$ in $U^{\prime}$.)

In this situation where $s^{\prime}<+\infty$, we may apply the local extension property of finely hyperharmonic functions [6, Theorem 9.9]. Let $x \in \varphi^{-1}\left(U^{\prime}\right)$ be given. There exists a finely open set $V^{\prime}$ with $^{3}$

$$
\varphi(x) \in V^{\prime} \subset \tilde{V}^{\prime} \subset U^{\prime}
$$

such that

$$
s^{\prime}=p^{\prime}-q^{\prime} \text { in } V^{\prime},
$$

where $p^{\prime}$ and $q^{\prime}$ are locally bounded potentials on $\Omega^{\prime}$, and $q^{\prime}$ is finely harmonic in $V^{\prime}$. According to [7, Theorem 4] there exists a compact, fine neighbourhood $K^{\prime}$ of $\varphi(x)$ with $K^{\prime} \subset V^{\prime}$, and a sequence of harmonic functions $u_{n}^{\prime}$ (each defined in some open set $\omega_{n}^{\prime} \subset \Omega^{\prime}$ with $\omega_{n}^{\prime} \supset K^{\prime}$ ), such that

$$
u_{n}^{\prime}\left|K^{\prime} \rightarrow q^{\prime}\right| K^{\prime} \quad \text { uniformly. }
$$

It follows that

$$
\left(u_{n}^{\prime} \circ \varphi\right)\left|V \rightarrow\left(q^{\prime} \circ \varphi\right)\right| V \quad \text { uniformly, }
$$

where $V$ denotes the fine interior of $\varphi^{-1}\left(K^{\prime}\right)$, which is a fine neighbourhood of $x$ in view of Property i) established above. By hypothesis, each

\footnotetext{
${ }^{3}$ Here and elsewhere the fine closure of a set $A$ in $\Omega$ or $\Omega^{\prime}$ is denoted by $\tilde{A}$.
} 
$\left(u_{n}^{\prime} \circ \varphi\right) \mid V$ is finely harmonic (in $V$ ), and hence so is $\left(q^{\prime} \circ \varphi\right) \mid V$ by virtue of [6, Lemma 9.6].

On the other hand $p^{\prime} \circ \varphi$ is finely hyperharmonic in $V$ by Lemma 4 , and we conclude that the function

$$
s^{\prime} \circ \varphi=p^{\prime} \circ \varphi-q^{\prime} \circ \varphi \quad(\text { in } V)
$$

is finely hyperharmonic in $V$, and consequently $s^{\prime} \circ \varphi$ is finely hyperharmonic in $\varphi^{-1}\left(U^{\prime}\right)$.

Corollary. Let $X$ and $X^{\prime}$ be finely open subsets of $\Omega$ and $\Omega^{\prime}$, respectively. If $\varphi: X \rightarrow \Omega^{\prime}$ and $\psi: X^{\prime} \rightarrow \Omega^{\prime \prime}$ are finely harmonic mappings, then so is $\psi \circ \varphi: X \rightarrow \Omega^{\prime \prime}$.

6. Th e o r e m. Suppose that the finely open set $X \subset \Omega$ is finely connected, and that $\varphi$ is a non-constant, finely harmonic mapping of $X$ into $\Omega^{\prime}$. Then $\varphi^{-1}\left(A^{\prime}\right)$ is polar ${ }^{4}$ in $\Omega$ for any polar set $A^{\prime}$ in $\Omega^{\prime}$.

Proof. In view of Lemma 4, the proof given in [2] for [2, Theorem 3.2] carries over to the present situation, using consistently the fine topology on $X$, but the initial topology on $\Omega^{\prime}$. One uses twice the fact that a finely hyperharmonic function in a fine domain ${ }^{5}$ is either identically $+\infty$ or else finite off a polar set [6, Theorem 12.9]. ${ }^{6}$ At the end it is proved that every point $x \in X$ has a finely open, fine neighbourhood $U_{x} \subset X$ such that $U_{x} \cap \varphi^{-1}\left(A^{\prime}\right)$ is polar. To conclude that $\varphi^{-1}\left(A^{\prime}\right)$ is itself polar, it remains only to apply Doob's quasi Lindelöf principle which shows that countably many $U_{x}$ cover $X$ up to a polar set.

As in [6], let us call a function finely superharmonic if it is finely hyperharmonic and moreover finite off some polar set. Then we have the following immediate corollary of Theorem 6 together with Property ii) in Theorem 5:

Corollary. Under the hypotheses of Theorem $6, s^{\prime} \circ \varphi$ is finely superharmonic in $\varphi^{-1}\left(U^{\prime}\right)$ for any finely superharmonic function $s^{\prime}$ in a finely open set $U^{\prime} \subset \Omega^{\prime} \quad\left(\right.$ such that $\left.\varphi^{-1}\left(U^{\prime}\right) \neq \varnothing\right)$.

7. $\mathrm{Th}$ e o r e m. Let $\varphi$ be a non-constant, finely harmonic mapping of a fine domain $X \subset \Omega$ into $\Omega^{\prime}$, and suppose that the points of $\varphi(X)$ are

${ }^{4}$ The notion of a polar set $A \subset \Omega$ (and similarly in $\Omega^{\prime}$ ) is taken in the local sense as in $[3, \S 6.2]$. If $\Omega$ (with a countable base) is $\mathfrak{P}$-harmonic, then $A$ is polar if and only if there exists a superharmonic function (even a potential) which equals $+\infty$ in $A$, see [3, Proposition 6.2.1 and Exercise 6.2.1] for the present general axiomatic frame.

5 By a fine domain is understood a finely connected, finely open set.

${ }^{6}$ A simpler proof of [6, Theorem 12.9] could easily be given, parallel to that of [3, Proposition 6.2.1]. 
polar (in $\Omega^{\prime}$ ). Then $\varphi(X)$ is finely open (in $\Omega^{\prime}$ ), and $\varphi$ is an open mapping with respect to the fine topologies on $X$ and $\Omega^{\prime}$.

Remark. In view of e) and a) in $\S 3$, this result contains [2, Theorem 3.5] in our case where $\Omega$ and $\Omega^{\prime}$ satisfy the domination axiom. Our proof is adapted from that of [2, Theorem 3.5], the main difference being that it is no longer possible to reduce to the case where the constants are harmonic (in both spaces $\Omega$ and $\Omega^{\prime}$ ). We shall therefore bring the complete proof.

Proof. Let $x \in X$ be given, and consider any fine neighbourhood $E$ of $x$ in $X$. We shall prove that $\varphi(E)$ is a fine neighbourhood of

$$
x^{\prime}:=\varphi(x)
$$

in $\Omega^{\prime}$. There exists a $\mathfrak{P}$-set $\omega^{\prime} \subset \Omega^{\prime}$ such that $x^{\prime} \in \omega^{\prime}$. We may suppose, in addition, that there exists a harmonic function $h^{\prime}$ on $\omega^{\prime}$ such that

$$
1 \leqq h^{\prime} \leqq 2 \text {. }
$$

Choose a $\mathfrak{P}$-set $\omega \subset \Omega$ so that $x \in \omega$. Since the fine topology is locally connected, it suffices to consider the case where $E$ is a fine domain contained in $\omega \cap \varphi^{-1}\left(\omega^{\prime}\right)$. According to Theorem $6, \varphi^{-1}\left(x^{\prime}\right)$ is polar. Since $E$ is non-polar and contains $x$, it follows that the restriction of $\varphi$ to $E$ is non-constant.

Replacing $\Omega$ by $\omega, \Omega^{\prime}$ by $\omega^{\prime}, X$ by $E$, and $\varphi$ by $\varphi \mid E$ (cf. a), §3), we may therefore assume from the beginning that $\Omega$ and $\Omega^{\prime}$ are $\mathfrak{B}$ harmonic spaces, and that $\Omega^{\prime}$ admits a harmonic function $h^{\prime}$ satisfying (2). And it is then our task to prove that $\varphi(X)$ is a fine neighbourhood of $x^{\prime}$.

According to Lemma 1 there exists a finely open set $U$ of compact closure $\bar{U}$ in $\Omega$ such that

$$
x \in U \subset \bar{U} \subset X,
$$

and such that $\varphi \mid \bar{U}$ is continuous in the initial topologies on $\bar{U}$ and $\Omega^{\prime}$. Replacing, if necessary, $U$ by the larger, finely open set $\mathrm{C} b(\mathrm{C} U)$, which has the same closure as $U$, we may further assume that $\mathrm{C} U(=\Omega \backslash U)$ is a base, that is, stable under the base operation $b .^{7}$

Being polar, $\varphi^{-1}\left(x^{\prime}\right)$ has measure 0 with respect to the generalized harmonic measure $\varepsilon_{x}^{C U}$ (the swept-out of the Dirac measure $\varepsilon_{x}$ onto C $U$ ) for any $x \in U .{ }^{8}$ Since $\varepsilon_{x}^{c U}$ is carried by $\partial_{f} U$, there exists accordingly a compact set $K \subset\left(\partial_{f} U\right) \backslash \varphi^{-1}\left(x^{\prime}\right)$ so large that

${ }^{7}$ For any set $A \subset \Omega$ (and similarly in $\Omega^{\prime}$ ), $b(A)$ is defined as the set of all points of $\Omega$ at which $A$ is not thin. It is a well-known consequence of axiom $D$ that the base operation $b$ is idempotent, and hence $b(A)$ is a base (for any set $A$ ).

${ }^{8} \mathrm{We}$ denote by $\partial_{f} U$ the fine boundary of $U$ (in $\Omega$ ). It is a well-known consequence of axiom $D$ that $\varepsilon_{x}^{\mathrm{C} U}$ is carried by $\partial_{f} U$ for any $x \in U$. - Incidentally, since $\varphi^{-1}\left(x^{\prime}\right)$ is polar and hence finely discrete we might choose $U$ above so that $\varphi^{-1}\left(x^{\prime}\right) \cap \bar{U}=\{x\}$, in particular $\varphi^{-1}\left(x^{\prime}\right) \cap \partial_{f} U=\varnothing$. 


$$
H_{f}^{U}(x)<\frac{1}{2}
$$

where $f$ denotes the indicator function for $\Omega \backslash K$, and where, by definition, for any $y \in \Omega$,

$$
H_{f}^{U}(y):=\int f d \varepsilon_{y}^{\mathrm{c} U}
$$

Since $K$ does not meet $\varphi^{-1}\left(x^{\prime}\right), x^{\prime}$ is not in $\varphi(K)$. Since $K \subset \bar{U}$, and $\varphi \mid \bar{U}$ is continuous, $\varphi(K)$ is compact in $\Omega^{\prime}$ (with the initial topology). Hence there exists a usual regular domain $U^{\prime}$ in $\Omega^{\prime}$ such that

$$
x^{\prime} \in U^{\prime} \subset \bar{U}^{\prime} \subset \mathrm{C} \varphi(K) \text {. }
$$

Essentially following [2], we write

$$
\begin{aligned}
& V:=U \cap \varphi^{-1}\left(U^{\prime}\right), \\
& G^{\prime}:=U^{\prime} \backslash \varphi(\bar{V}) .
\end{aligned}
$$

Then $V$ is a finely open subset of $X$ containing $x$, being the intersection of two such sets. And $G^{\prime}$ is open in $\Omega^{\prime}$ because $U^{\prime}$ is open, and $\varphi(\bar{V})$ is compact (since $\bar{V}$ is a compact subset of $\bar{U}$, and $\varphi \mid \bar{U}$ is continuous). Note also that

$$
V \subset \varphi^{-1}\left(C G^{\prime}\right)
$$

by (8), and further that $\bar{V} \subset X$ in view of (3), (7). Consequently, we obtain from (8)

$$
U^{\prime} \backslash G^{\prime} \subset \varphi(\bar{V}) \subset \varphi(X),
$$

and it is therefore sufficient to prove that $G^{\prime}$ is thin at $x^{\prime}$.

Let $K^{\prime}$ denote an arbitrary compact subset of $G^{\prime}$, and write, as in [2],

$$
s^{\prime}:=\left\{\begin{aligned}
\hat{R}_{h^{\prime}}^{K^{\prime}} & \text { in } U^{\prime} \\
0 & \text { in } \Omega^{\prime} \backslash U^{\prime}
\end{aligned}\right.
$$

where the swept-out $\hat{R}_{h^{\prime}}^{K^{\prime}}$ is understood relatively to the harmonic subspace $U^{\prime}$ of $\Omega^{\prime}$. Since $U^{\prime}$ is regular, it is well known that $s^{\prime}$ is continuous at every point of $\Omega^{\prime} \backslash K^{\prime}$. Moreover, $s^{\prime}$ is finely continuous in $U^{\prime}$ (being hyperharmonic there), and so $s^{\prime}$ is finely continuous in all of $\Omega^{\prime}$. Hence $s^{\prime} \circ \varphi$ is finely continuous in $X$ by Property i) in Theorem 5 .

The function

$$
u:=H_{f}^{U}-\frac{1}{2} s^{\prime} \circ \varphi
$$

is well defined $(<+\infty)$ and finely lower semicontinuous in $X$. In fact, $H_{f}^{U}$ is finite and finely lower semicontinuous in $\Omega$ according to [6, Lemma 
9.12] applied to the base $\Omega \backslash U$ in $\Omega$, noting that $f$ is (finely) lower semicontinuous. ${ }^{9}$ The same lemma shows that $H_{t}^{U}$ is finely harmonic in $U$, and hence in $V$. And $s^{\prime} \circ \varphi$ is likewise finely harmonic in $V$ because $s^{\prime}$ is harmonic in $U^{\prime} \backslash K^{\prime}$ by (10), and $V \subset \varphi^{-1}\left(U^{\prime} \backslash K^{\prime}\right)$ according to (7) and (9) and the fact that $K^{\prime} \subset G^{\prime}$.

We have thus found that $u$ is finely harmonic in $V$, and finely lower semicontinuous in $X$. Moreover $u \geqq-1$ in $X$ because $H_{f}^{U} \geqq 0$ and

$$
s^{\prime} \circ \varphi \leqq h^{\prime} \circ \varphi \leqq 2
$$

by virtue of (2). According to the fine boundary minimum principle $[6$, Theorem 9.1], the conclusion

$$
u \geqq 0 \quad \text { in } V
$$

can be drawn if we can show that $u(y) \geqq 0$ for every $y \in \partial_{f} V$. (Note that the fine closure $\tilde{V}$ of $V$ is contained in $X$ according to (7) and (3).)

From (7) it follows that

$$
\partial_{f} V \subset\left(\partial_{f} U\right) \cup\left(\partial_{f} \varphi^{-1}\left(U^{\prime}\right)\right) .
$$

Note also that

$$
\partial_{f} V \subset \mathrm{CK}
$$

on account of (6) because

$$
\partial_{f} V \subset \tilde{V} \subset \varphi^{-1}\left(\tilde{U}^{\prime}\right) \subset \varphi^{-1}(\subset \varphi(K)) \subset \subset K .
$$

If $y \in \partial_{f} U$, then $y \in\left(\partial_{f} U\right) \backslash K$ by (15), and hence $f(y)=1$. Since $\mathrm{C} U$ is a base, we have $\varepsilon_{y}^{\mathrm{CU}}=\varepsilon_{y}$, and consequently

$$
H_{f}^{U}(y)=\int f d \varepsilon_{y}^{C U}=f(y)=1 .
$$

When combined with (11) and (12), this yields $u(y) \geqq 0$ (in the case $\left.y \in \partial_{f} U\right)$.

In the remaining case $y \in \partial_{f} \varphi^{-1}\left(U^{\prime}\right)$, cf. (14), we have $\varphi(y) \in \partial_{f} U^{\prime}$ (since $\varphi^{-1}\left(U^{\prime}\right)$ is finely open), and hence

$$
\left(s^{\prime} \circ \varphi\right)(y)=s^{\prime}(\varphi(y))=0
$$

by (10) (since $U^{\prime}$ is finely open). It follows again that $u(y) \geqq 0$.

Having thus established (13), we complete the proof by showing that $G^{\prime}$ is indeed thin at $x^{\prime}$. Since $\varphi(x)=x^{\prime} \in U^{\prime}$ by (6), we have from (10)

$$
\left(s^{\prime} \circ \varphi\right)(x)=\hat{R}_{h^{\prime}}^{K^{\prime}}\left(x^{\prime}\right) \text {. }
$$

${ }^{9} \mathrm{In}[6], H_{f}^{U}$ is denoted instead by $f^{\mathrm{CU}}$. Since $\Omega$ is $\mathfrak{P}$-harmonic, there exists a locally bounded potential $p>0$ on $\Omega$. We may assume that $p \geqq 1(\geqq f)$ in $\bar{U}$. 
Since $u(x) \geqq 0$, we obtain from (11)

$$
\hat{R}_{h^{\prime}}^{K^{\prime}}\left(x^{\prime}\right) \leqq 2 H_{f}^{U}(x),
$$

and hence, by varying $K^{\prime}$, using e.g. [3, Corollary 4.2.2],

$$
\hat{R}_{h^{\prime}}^{G^{\prime}}\left(x^{\prime}\right) \leqq 2 H_{f}^{U}(x)<1 \leqq h^{\prime}\left(x^{\prime}\right)
$$

on account of (4) and (2). This shows that $G^{\prime}$ is thin at $x^{\prime}$, and the proof is complete.

Remark. Instead of supposing that the points of $\varphi(X)$ are polar, it would clearly suffice to assume that $\varphi^{-1}\left(\left\{x^{\prime}\right\}\right)$ is a polar subset of $\Omega$ for every non-polar point $x^{\prime} \in \varphi(X)$ (and hence actually for any $x^{\prime} \in \Omega^{\prime}$ by Theorem 6). As in the case of harmonic mappings, some restriction of this kind is, however, needed, as it appears from an example due to A. Cornea (personal communication). To illustrate the role of non-polar points in $\Omega^{\prime}$ note also that (corresponding to the case $\Omega^{\prime}=\mathbf{R}$, ef. b) of $\S 3$ ) it seems to be an open problem whether, say in the case $\Omega=\mathbf{R}^{n}, n \geqq 2$, a nonconstant finely harmonic function $u$ in a fine domain $X \subset \Omega$ is nonconstant in every non-void finely open subset of $X$. (This would of course follow from Theorem 7 above if the hypothesis concerning non-polarity of points could be dropped.)

8. The or e m. Let $\varphi: X \rightarrow \Omega^{\prime}$ be an injective, finely harmonic mapping of a finely open set $X \subset \Omega$ into $\Omega^{\prime}$. Suppose that the points of one of the sets $X$ or $X^{\prime}:=\varphi(X)$ are polar. Then so are the points of the other set $\left(X^{\prime}\right.$ or $\left.X\right)$. Moreover, $X^{\prime}$ is finely open, and $\varphi^{-1}: X^{\prime} \rightarrow \Omega$ is a finely harmonic mapping.

Proof. As in the beginning of the proof of the preceding theorem we may easily reduce to the case where $\Omega$ and $\Omega^{\prime}$ are $\mathfrak{P}$-harmonic spaces and where $X$ and (hence) $X^{\prime}$ are finely connected. According to Theorem $7, \varphi$ is a finely open mapping, hence altogether a fine homeomorphism of $X$ onto $X^{\prime}$. In particular, $X^{\prime}$ is finely open in $\Omega^{\prime}$, and in fact a fine domain in the present case.

Let $u$ be finely harmonic in a finely open set $U \subset X$. In order to prove that $u \circ \varphi^{-1}$ is finely harmonic in the finely open set $\varphi(U) \subset X^{\prime}$, we proceed as in the proof of [2, Theorem 3.4]. According to Lemma 1 (applied to $\varphi^{-1}$ ) there exists for any $x^{\prime} \in \varphi(U)$ a finely open set $V^{\prime}$ of compact closure $\vec{V}^{\prime}$ in $\Omega^{\prime}$ such that

$$
x^{\prime} \in V^{\prime} \subset \bar{V}^{\prime} \subset \varphi(U),
$$

and such that $\varphi^{-1} \mid \bar{V}^{\prime}$ is continuous in the initial topologies on $\bar{V}^{\prime}$ and $\Omega$. As in connection with (3) in the proof of Theorem 7, we may further arrange 
that $\Omega^{\prime} \backslash V^{\prime}$ is a base (in $\Omega^{\prime}$ ). Finally we may assume that $u \circ \varphi^{-1}$ is bounded on $\bar{V}^{\prime}$. (Note that $u \circ \varphi^{-1}$ is finely continuous and finite in $\varphi(U)$.) Since $\Omega^{\prime}$ is $\mathfrak{P}$-harmonic, there exists a locally bounded potential $p^{\prime}>0$ on $\Omega^{\prime}$. We may assume that $\left|u \circ \varphi^{-1}\right| \leqq p^{\prime}$ in $\overline{V^{\prime}}$.

Using again the notation introduced in (5) above, but now in the space $\Omega^{\prime}$, we put

$$
v^{\prime}:=H_{u \circ \varphi^{-1}}^{V^{\prime}} .
$$

According to [6, Lemma 9.3] $v^{\prime}$ is well-defined, bounded, and finely continuous in $\tilde{V}^{\prime}$, and finely harmonic in $V^{\prime}$. Clearly $v^{\prime}$ agrees with $u \circ \varphi^{-1}$ on $\partial_{f} V^{\prime}\left(\subset \varphi(U) \backslash V^{\prime}\right)$.

Hence it follows from Theorem 5 that $v^{\prime} \circ \varphi$ is bounded and finely continuous in the fine closure $\varphi^{-1}\left(\tilde{V}^{\prime}\right)$ of $\varphi^{-1}\left(V^{\prime}\right)$, and finely harmonic in $\varphi^{-1}\left(V^{\prime}\right)$ itself. Moreover, $v^{\prime} \circ \varphi=u$ on $\partial_{f} \varphi^{-1}\left(V^{\prime}\right)=\varphi^{-1}\left(\partial_{f} V^{\prime}\right)$. Since $\varphi^{-1}\left(V^{\prime}\right)$ is contained in the compact subset $\varphi^{-1}\left(\bar{V}^{\prime}\right)$ of $\Omega$, it follows by application of the fine boundary minimum principle $[6, \S 9.1]$ that $v^{\prime} \circ \varphi=u$ in $\varphi^{-1}\left(V^{\prime}\right)$, that is, $v^{\prime}=u \circ \varphi^{-1}$ in $V^{\prime}$, and consequently $u \circ \varphi^{-1}$ is indeed finely harmonic in $V^{\prime}$. - The assertion concerning polarity of points follows from Theorem 6 applied to $\varphi^{-1}$ or $\varphi$.

9. The o r e m. Let $\varphi: X \rightarrow \Omega^{\prime}$ be a finely harmonic mapping, and let $X^{\prime}$ denote a non void, fine domain in $\Omega^{\prime}$ such that $\varphi(X) \subset X^{\prime}$. If there exists a finite, fine potential $p^{\prime}>0$ on $X^{\prime}$ such that $p^{\prime} \circ \varphi$ is a fine potential on $X$, then $X^{\prime} \backslash \varphi(X)$ is inner polar.

Proof. Let $K^{\prime}$ denote a compact subset of $X^{\prime} \backslash \varphi(X)$, and write

$$
s^{\prime}:=\hat{R}_{p^{\prime}}^{K^{\prime}} \text {, relative to } X^{\prime},
$$

see $[6, \S 11.4]$. Then $s^{\prime}(\geqq 0)$ is finely harmonic and $\leqq p^{\prime}$ in $\varphi(X)$ $\left(\subset X^{\prime} \backslash K^{\prime}\right)$ according to [6, Corollary 11.13]. Hence, by Theorem 5, $s^{\prime} \circ \varphi$ is finely harmonic, and of course $\leqq p^{\prime} \circ \varphi$ in $X$. By hypothesis, $p^{\prime} \circ \varphi$ is a fine potential on $X$ [6, Definition 10.5], and consequently $s^{\prime} \circ \varphi=0$ in $X$, that is $s^{\prime}=0$ on $\varphi(X)$. Since $X^{\prime}$ is finely connected, it follows from [6, Theorem 12.6] that $s^{\prime}=0$ in all of $X^{\prime}$, and so $K^{\prime}$ is indeed polar according to [6, Theorem 11.8] (applied to $f:=p^{\prime} \cdot 1_{K^{\prime}}$ on $\left.X^{\prime}\right)$.

Remark. Instead of assuming that $X^{\prime}$ is a fine domain it would suffice to suppose that every fine component of the finely open set $X^{\prime}$ (containing $\varphi(X))$ in $\Omega^{\prime}$ meets $\varphi(X)$. And instead of assuming that $p^{\prime}>0$ be a finite, fine potential on $X^{\prime}$ it is of course enough to suppose that $p^{\prime}>0$ is finely hyperharmonic and finite in $X^{\prime}$. The finiteness of $p^{\prime}$ can be dropped if $\Omega^{\prime}$ is $\mathfrak{B}$-harmonic. 
10. Approximation by harmonic mappings. Let $d^{\prime}$ denote a fixed metric on $\Omega^{\prime}$, compatible with the initial topology on $\Omega^{\prime}$.

$\mathrm{L}$ e m m a. If a net $\left(\varphi_{j}\right)_{j \in J}$ of finely harmonic mappings $\varphi_{j}: X \rightarrow \Omega^{\prime}$ converges pointwise to a mapping $\varphi: X \rightarrow \Omega^{\prime}$, and if every point of $X$ has a fine neighbourhood on which the convergence is uniform with respect to $d^{\prime}$, then the limit mapping $\varphi$ is a finely harmonic mapping of $X$ into $\Omega^{\prime}$.

Proof. Clearly $\varphi$ satisfies the continuity condition i) in Definition 2. Now let us check condition ii). For given $x \in X$ let $u^{\prime}$ be harmonic in some open neighbourhood $U^{\prime}$ of $\varphi(x)$ in $\Omega^{\prime}$. Let $B^{\prime}$ and $b^{\prime}$ denote compact $d^{\prime}$-balls with centre $\varphi(x)$ such that $b^{\prime} \subset B^{\prime} \subset U^{\prime}$ (and such that the radius of $b^{\prime}$ is smaller than that of $\left.B^{\prime}\right)$. By hypothesis there is a fine neighbourhood $V$ of $x$ in $X$ such that $\varphi(V) \subset b^{\prime}$ and $\varphi_{j} \mid V \rightarrow$ $\varphi \mid V \quad d^{\prime}$-uniformly. It follows that $\varphi_{j}(V) \subset B^{\prime}$ for all $j$ from a certain step. Since $u^{\prime}$ is uniformly continuous on $B^{\prime}$, we conclude that

$$
\left(u^{\prime} \circ \varphi_{j}\right)\left|V \rightarrow\left(u^{\prime} \circ \varphi\right)\right| V \quad \text { uniformly, }
$$

and so $u^{\prime} \circ \varphi$ is finely harmonic in $V$ by [6, Lemma 9.6].

$\mathrm{D}$ efinition. For any compact set $K \subset \Omega$ let $H\left(K, \Omega^{\prime}\right)$ denote the $d^{\prime}$-uniform closure of the set of all restrictions to $K$ of harmonic mappings of open neighbourhoods of $K$ (in $\Omega$ ) into $\Omega^{\prime}$.

Corollary. A sufficient condition for a mapping $\varphi: X \rightarrow \Omega^{\prime}$ to be finely harmonic is that every point $x \in X$ has a compact, fine neighbourhood $K \subset X$ such that $\varphi \mid K \in H\left(K, \Omega^{\prime}\right)$.

This follows from the above lemma applied to the fine interior $V$ of $K$ since the restriction to $V$ of any harmonic mapping of an open neighbourhood of $K$ into $\Omega^{\prime}$ is a finely harmonic mapping of $V$ into $\Omega^{\prime}$ according to e) and a), §3.

Problem. Is the condition in the above corollary also necessary in order that $\varphi$ be a finely harmonic mapping?

According to [7, Theorem 4] the answer to this question is affirmative in the very particular case $\Omega^{\prime}=\mathbf{R}$, cf. b), $\S 3$.

The problem seems to be a difficult one even for $\Omega=\Omega^{\prime}=\mathbf{R}^{2}$ (with the classical harmonic sheaf).

11. Finely holomorphic functions. In this final section we shall specialize to the case

$$
\Omega=\Omega^{\prime}=\mathbf{C},
$$

the complex plane endowed with the classical harmonic sheaf on $\mathbf{C}=\mathbf{R}^{2}$.

Definition. Let $X$ denote a finely open subset of C. A function $\varphi: X \rightarrow \mathbf{C}$ is called finely holomorphic if every point $z \in X$ has a compact, fine neighbourhood $K \subset X$ such that $\varphi \mid K \in R(K)$. 
Here $R(K)$ denotes the uniform closure of the algebra of all restrictions to $K$ of rational functions on $\mathbf{C}$ with poles off $K$, or equivalently of all restrictions to $K$ of holomorphic functions in open neighbourhoods of $K$ in C. See, e.g., Zalcman [9].

Clearly the finely holomorphic functions in $X$ form a subalgebra of the algebra of all finely continuous, complex valued functions in $X$. Moreover they have, in their dependence on $X$, the sheaf property analogous to a), §3.

Th e o r e m. Let $\varphi: X \rightarrow \mathbf{C}$ be finely holomorphic (or the conjugate of a finely holomorphic function). Then $\varphi$ is a finely harmonic mapping of $X$ into C. If $X$ is finely connected and if $\varphi$ is non-constant, then $\varphi$ is a finely open mapping, and the zeros of $\varphi$ form a polar (= finely discrete) set.

Proof. Clearly every holomorphic (or antiholomorphic) function in an open set $\omega \subset \mathbf{C}$ is a harmonic mapping of $\omega$ into $\mathbf{C}$. In view of e) and a), $\S 3$, it therefore follows from Lemma 10 (taking for $d^{\prime}$ the usual metric on $\Omega^{\prime}=\mathrm{C}$ ) that $\varphi$ is a finely harmonic mapping. (Alternatively, use c), §3.) The remaining assertions are therefore contained in Theorems 7 and 6 , respectively.

Remarks. a) It is easily shown that if $\varphi$ and $\psi$ are finely holomorphic in the finely open sets $X$ and $X^{\prime}$, respectively, then $\psi \circ \varphi$ is finely holomorphic in $\varphi^{-1}\left(X^{\prime}\right)$. (Consider first the case where $X^{\prime}$ is open and $\psi$ is holomorphic.)

b) If $\varphi$ and $\bar{\varphi}$ are both finely holomorphic in a fine domain $X$, then $\varphi$ is constant. (In fact, $\operatorname{Re} \varphi$ and $\operatorname{Im} \varphi$ must be finely holomorphic, too, and hence constant since their ranges have no finely interior points in $\mathbf{C}$.)

c) A function $\varphi$ defined in a usual open set $\omega \subset \mathbf{C}$ is finely holomorphic there if and only if it is holomorphic. (As to the non-trivial "only if" part, say in the case where $\omega$ is connected, and hence finely connected by [6, Corollary 9.8], note that $\varphi$ is a harmonic mapping by the above theorem together with e), §3. In particular, $\varphi$ and $\varphi^{2}$ are complex, harmonic functions in $\omega$, and this implies that $\varphi$ is conformal, that is, either $\varphi$ or $\bar{\varphi}$ is holomorphic [as a function of the complex variable $z \in \mathbf{C}$ ]. The latter alternative cannot occur according to the preceding remark b), except in the trivial case of a constant function.)

In [4], Debiard and Gaveau have introduced an interesting, possibly larger sheaf of algebras of generalized holomorphic functions with respect to the fine topology on $\mathbf{C}$. For any finely open set $X \subset \mathbf{C}$ they denote by $O(X)$ the class of all complex, finely harmonic functions $\varphi: X \rightarrow \mathbf{C}$ such that, roughly speaking, $\partial \varphi / \partial \bar{z}=0$ in $X$ in the sense of stochastic differentiation along the Brownian paths. 
Using Lemma 1 above, it follows from the results in [4] that $O(X)$ is an algebra, closed in the sense corresponding to Lemma 10 above, and hence containing all finely holomorphic functions as defined at the outset of the present section. Moreover, [4] brings the first proof that every non-constant function of class $O(X)$ is a finely open mapping (if $X$ is a fine domain).

According to c), §3, every function $\varphi \in O(X)$ is a finely harmonic mapping of $X$ into $\mathbf{C}$ (and so is $\bar{\varphi}$ ). Thus the above theorem extends to the class $O(X)$, and likewise Remarks b) and c) above (with similar proofs).

Problem. Let $\varphi$ be a finely harmonic mapping of a fine domain $X \subset \mathbf{C}$ into $\mathbf{c}$. Does it follow that either $\varphi$ or $\bar{\varphi}$ is of class $O(X)$ (or even finely holomorphic)?

In its latter, stronger form, this problem is equivalent to the problem stated at the end of $\$ 10$ (in the present case $\Omega=\Omega^{\prime}=\mathrm{C}$ ).

\section{References}

[1] BRELOT, M.: Recherches axiomatiques sur un théorème de Choquet concernant l'effilement. - Nagoya Math. J. 30, 1967, 39-46.

[2] Constantinescu, C., and A. Cornea: Compactifications of harmonic spaces. Nagoya Math. J. 25, 1965, 1-57.

[3] -»- -»- Potential theory on harmonic spaces. - Die Grundlehren der mathematischen Wissenschaften 158. Springer-Verlag, Berlin - Heidelberg - New York, 1972.

[4] DeBIARD, A., and B. GRAvEAU: Potentiel fin et algèbres de fonctions analytiques II. - J. Functional Analysis 17, 1974, 296-310.

[5] FUGLEDE, B.: The quasi topology associated with a countably subadditive set function. - Ann. Inst. Fourier (Grenoble) 21:1, 1971, 123-169.

[6] -»- Finely harmonic functions. - Lecture Notes in Mathematics 289. SpringerVerlag, Berlin - Heidelberg - New York, 1972.

[7] - - Fonctions harmoniques et fonctions finement harmoniques. - Ann. Inst. Fourier (Grenoble) 24: 4, 1974, 77-91.

[8] LaIne, I.: Covering properties of harmonic Bl-mappings II. - Ann. Acad. Sci. Fenn. Ser. A.I. 570, 1974.

[9] ZaLCMAN, L.: Analytic capacity and rational approximation. - Lecture Notes in Mathematics 50. Springer-Verlag, Berlin - Heidelberg - New York, 1968.

Københavns Universitet

Matematisk Institut

Universitetsparken 5

DK-2100 København Ø

Denmark

Received 14 February 1975 